\title{
Morphological and cell kinetic effects of dietary manipulation during colorectal carcinogenesis
}

\author{
D J GALLOWAY, FREDA JARRETT, P BOYLE, MALLIKA INDRAN, \\ KATHARINECARR, R W OWEN, AND W D GEORGE
}

From the University Departments of Surgery, Pathology and Anatomy, Western Infirmary and University of Glasgow, Glasgow, and Bacterial Metabolism Research Laboratory, Centre for Applied Microbiology and Research PHLS Porton Down, Salisbury, Wilts.

SUMmaRy The effect of dietary manipulation of fat and fibre on the structural and cell kinetic characteristics of colonic mucosa was studied before and during experimental carcinogenesis in 232 male Albino Swiss rats. Carcinogen treated animals were given 12 weekly injections of azoxymethane $(10 \mathrm{mg} / \mathrm{kg} /$ week $)$. The animals were divided between four dietary groups (1) high fat, high fibre, (2) low fat, high fibre, (3) high fat, low fibre and (4) low fat, low fibre. Pathological and cell kinetic information together with details of certain faecal characteristics was collected when the animals were killed 4,20, and 28 weeks after starting their experimental diet. Tumour induction was significantly influenced by diet. The highest risk of colorectal tumour development was found in groups fed diet 3: high fat, low fibre $(\mathrm{p}<0.03)$. In contrast, diet 2: low fat, high fibre was associated with the lowest risk. The proportion of histologically proven colonic tumours occuring in each dietary group was: diet $1-10 \cdot 9 \%$, diet $2-3 \cdot 6 \%$, diet $3-63 \cdot 7 \%$, diet $4-21 \cdot 8 \%$. Scanning electron microscopic (SEM) studies done on selected samples indicated both dietary and azoxymethane related alterations in crypt unit integrity. The most marked surface architectural changes were seen in carcinogen treated animals maintained on diet 3 (high fat, low fibre). Stathmokinetic analysis revealed considerable intergroup variability. Both fat and fibre produced significant effects, principally during the preneoplastic phase of carcinogenesis. Faster proliferative activity tended to be found in animals at low risk of tumour induction (diet 2), slower proliferation being more characteristic of animals at high risk $(p<0.05)$ The findings suggest that both topographical and cell kinetic parameters have an important relationship with promoting and protecting dietary factors during the development of colorectal cancer.

Colorectal cancer is at present the second most frequent cause of cancer related death in the Western World. Despite increasing sophistication in both diagnostic and therapeutic strategies, little improvement has been evident in the outcome after management of this condition. Much of the current research relative to large bowel cancer is thus being directed towards achieving a clearer understanding of the aetiological and biological properties of the disease.

Descriptive and analytical epidemiological studies $^{1-4}$ have, by highlighting the marked international incidence variation for colorectal cancer,

Address for correspondence: Mr David J Gallowaly. Dept of Surgery. Western Infirmary. Glasgow G11 6NT.

Received for publication 3 October 1986 consistently emphasised the importance of environmental factors in the aetiology of this condition. In particular, dietary factors appear to be important, especially dietary fat and fibre which have been shown to have promoting and protecting influences respectively.

Over the past decade several good animal models for experimental colorectal cancer have been developed and investigated. Wide experience has been gained with a particularly reliable model which involves the induction of large bowel cancer in rodents using such hydrazine carcinogens as 1, 2dimethyl-hydrazine or azoxymethane. manipulation of fat and fibre during experimental carcinogenesis has shown respective promoting and protecting influences attributable to these crude nutrients in the animal model. ${ }^{5}$ 
In the process of neoplastic transformation it is clear that functional disturbances in the proliferative characteristics of any tissue undergoing neoplastic change are required before the development of the recognisable morphological abnormalities that constitute neoplasia. Current understanding of the histogenesis of colorectal cancer owes much to morphological studies. ${ }^{67}$ Until recently, however, there has been relatively little emphasis on the study of the functional changes in growth characteristics which can account for the resulting histological pattern. ${ }^{.}$Thus kinetic parameters demand scrutiny in any serious study of the histogenesis of colorectal cancer.

It is clear, therefore, that both dietary factors and cell kinetic characteristics bear a fundamental relationship to the development of colorectal cancer. The purpose of this study was to examine and characterise the effects of the manipulation of dietary fat and fibre on the structural and cell kinetic characteristics of colonic mucosa during the induction of experimental large bowel cancer.

\section{Methods}

\section{STUDY DESIGN}

The experimental system used in this study involved the induction of colorectal cancer in inbred, male Albino Swiss rats using subcutaneous azoxymethane given in a dose of $10 \mathrm{mg} / \mathrm{kg} /$ week for 12 consecutive weeks. The animals were $8-12$ weeks old at the start of the experiment and they were housed in cages with stainless steel grid floors to minimise coprophagia. Two hundred and thirty two animals were studied and divided between four dietary groups. Both the experimental diets and water were available to the animals ad libitum. The diets were commercially prepared to the crude nutrient specifications shown in Table 1. (Special Diet Services, Witham, Essex).

Table 1 Dietary composition; crude nutrient content (adjusted to $10 \%$ water content)

\begin{tabular}{lcccc}
\hline & $\begin{array}{c}\text { Diet 1 } \\
\text { High fat } \\
\text { high fibre }\end{array}$ & $\begin{array}{c}\text { Diet 2 } \\
\text { Low fat } \\
\text { high fibre }\end{array}$ & $\begin{array}{c}\text { Diet 3 } \\
\text { High fat } \\
\text { low fibre }\end{array}$ & $\begin{array}{c}\text { Diet 4 } \\
\text { Low fat } \\
\text { low fibre }\end{array}$ \\
\hline Moisture (\%) & 10 & 10 & 10 & 10 \\
Crude fat (\%) & $25 \cdot 1$ & $2 \cdot 1$ & $25 \cdot 1$ & 1.9 \\
Crude fibre (\%) & $26 \cdot 6$ & $26 \cdot 9$ & $2 \cdot 1$ & 1.9 \\
Crude protein (\%) & 14.9 & $14 \cdot 8$ & 14.9 & $14 \cdot 7$ \\
Carbohydrate (\%) & $16 \cdot 4$ & $38 \cdot 7$ & $43 \cdot 7$ & $67 \cdot 2$ \\
Ash & $7 \cdot 0$ & $7 \cdot 5$ & $4 \cdot 1$ & $4 \cdot 3$ \\
Total (\%) & 100 & 100 & 100 & 100 \\
Digestive energy & 13.0 & $8 \cdot 2$ & 17.9 & 13.2 \\
& & 14.7 & $20 \cdot 3$ & 14.9 \\
\hline
\end{tabular}

Each diet contained the same standard vitamin and mineral supplementation. The source of fat in the diets was beef tallow and the principal source of fibre was purified cellulose. Morphological and cell kinetic observations were made at three different stages of carcinogenesis as follows:

1 0/12 category

The animals in this measurement category were maintained on the respective diets for four weeks before being killed. No carcinogen or control injections were administered.

\section{4/12 category}

Animals in this group were maintained on their respective diet for four weeks and then, in addition, a course of control or carcinogen injections were given and the animals killed four months from the time of the first injection.

3 6/12 category

In this group the animals were treated in the same way as the $4 / 12$ category except that the time of death was six months after the first injection.

GROUP SIZE

In the $0 / 12$ category each of the four dietary groups contained 10 animals. In both the $4 / 12$ and $6 / 12$ categories both control and carcinogen treated groups for each of the four diets comprised 12 animals.

SAMPLING PROCEDURE

At the time of death an identical sampling procedure was followed for each group of up to 12 animals. Eight of these animals were used for the stathmokinetic analysis. These animals received intraperitoneal vincristine $(1 \mathrm{mg}$ per $\mathrm{kg})$ at $900 \mathrm{am}$ on the day of study. Individual animals within the group were then killed at carefully defined time intervals $(30,45,60,75,90,105,120$, and 180 minutes) over the ensuing three hours. ${ }^{9}$ All of these animals were then subjected to the same post mortem examination. The gastrointestinal tract was excised and opened along its length. Standard full thickness specimens were taken from the colon at four defined sites corresponding to the rectum, the descending colon, the major flexure and the caecum. Two corresponding samples were taken from each of these four areas and were processed for histology and cell population kinetic analysis respectively. The liver was also removed from each animal and was processed for histology as was any other abnormal feature detected on autopsy.

HISTOLOGY

The tissue samples were fixed in buffered $10 \%$ formalin. Dehydration and blocking in paraffin wax was carried out and four micron thick sections were 
cut and then stained using a regressive haemalum and eosin staining technique before examination. Standard histological sections from three animals in each individual subgroup - that is, 20 subgroups, and from each of the four anatomical sites were examined for evidence of crypt hyperplasia by carefully counting the nuclei along the length of 10 axially sectioned crypts on each slide.

\section{SCANNING EIECTRON MICROSCOPY}

Paraffin embedded tissue blocks from the descending colon of each dietary group studied in the 4/12 category were taken and reprocessed for scanning electron microscopy by removing the paraffin wax from the tissue firstly by trimming and subsequently by melting in a pan of hot wax. The samples were then taken through four changes of xylene and then two changes each of absolute alcohol and amyl acetate before critical point drying with carbon dioxide. They were then mounted on stubs using silver paint, coated with gold in a sputter coater and examined in a JEOL T300 scanning electron micro- scope at $20 \mathrm{KV}$ without any stage tilt."' The recording was done on FP4 $70 \mathrm{~mm}$ films by exposing it for 90 seconds.

To facilitate the analysis of the SEM appearances a scoring system was used. This was developed from a previously validated system for the assessment of villous damage in irradiated small intestinal mucosa."

The scoring system is as follows: (): Spherical or ovoid crypt units with round or ovoid crypt orifices; normal appearance. (Fig. 1), 2: Elongated crypt units with slit like orifices, 4 : Large crypt units with wide orifices, 6: Crypt units showing wide orifices with swollen edges, 8: Absence of definitive crypt units (Fig. 2), 10: Total absence of epithelium.

When a sample contained crypt units fitting more than one of the descriptive categories, an average of the individual total score for each dietary group was calculated.

CEILI KINETICS

The tissue samples for kinetic analysis were fixed in Carnoy's fluid for six hours, rehydrated, hydrolysed

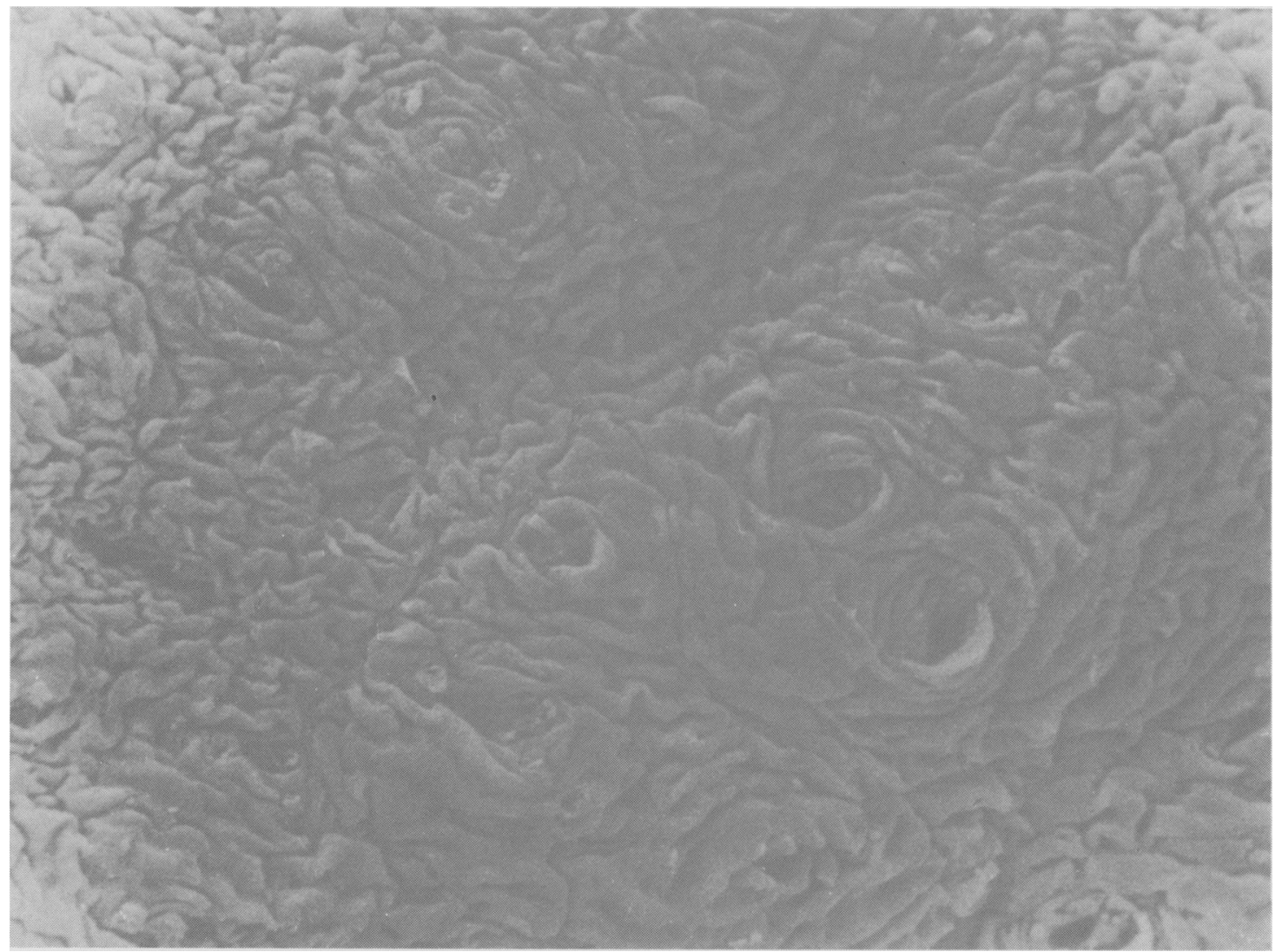

Fig. 1 Normal scanning electron microscopic appearances. Crypt units are spherical or ovoid with round or ovoid crypt orifices; a crypt unit integrity score of zero was given. 
in normal hydrochloric acid at $60^{\circ} \mathrm{C}$ for 10 minutes before staining in Schiffs reagent for one hour. The mucosa was then microdissected and a squashed preparation of whole colonic crypts was obtained which was then mounted in clear epoxy resin. Mean whole crypt metaphase counts from 10 crypts in each sample were then plotted against time to derive the crypt cell production rate by linear regression analysis.

\section{STATISTICAL ANALYSIS}

In order to make estimates of the independent effects of the different levels of dietary fat and fibre intakes as well as the administration of a carcinogen, the use of a multivariate analysis was required. This was accomplished using the computer package GLIM'2 (Generalised Linear Interactive Modelling). In order to test whether the crypt cell production rates differed between the dietary groups a modified linear regression analysis was used. Because each point on any slope was calculated from a number of observations and the standard errors of each point varied considerably, it was necessary to perform the regression 'weighting' each point inversely according to its standard error. This avoided the situation where one very high or low aberrant value based on few observations could over-influence the calculated slope.

FAECAI. CHARACTERISTICS

The weight of faeces produced by animals in the four dietary groups within the $4 / 12$ category was recorded during two separate seven day periods. These periods were located during week six and week 16, - that is, half way through the course of injections and immediately before death.

Faeces from each animal were tested just before death for the presence of occult blood using a modified guiac test. (Haemoccult; Eaton Labs).

The identification and quantification of faecal steroids was done as previously described. ${ }^{1:}$ Faecal samples from each group were pooled and extracts

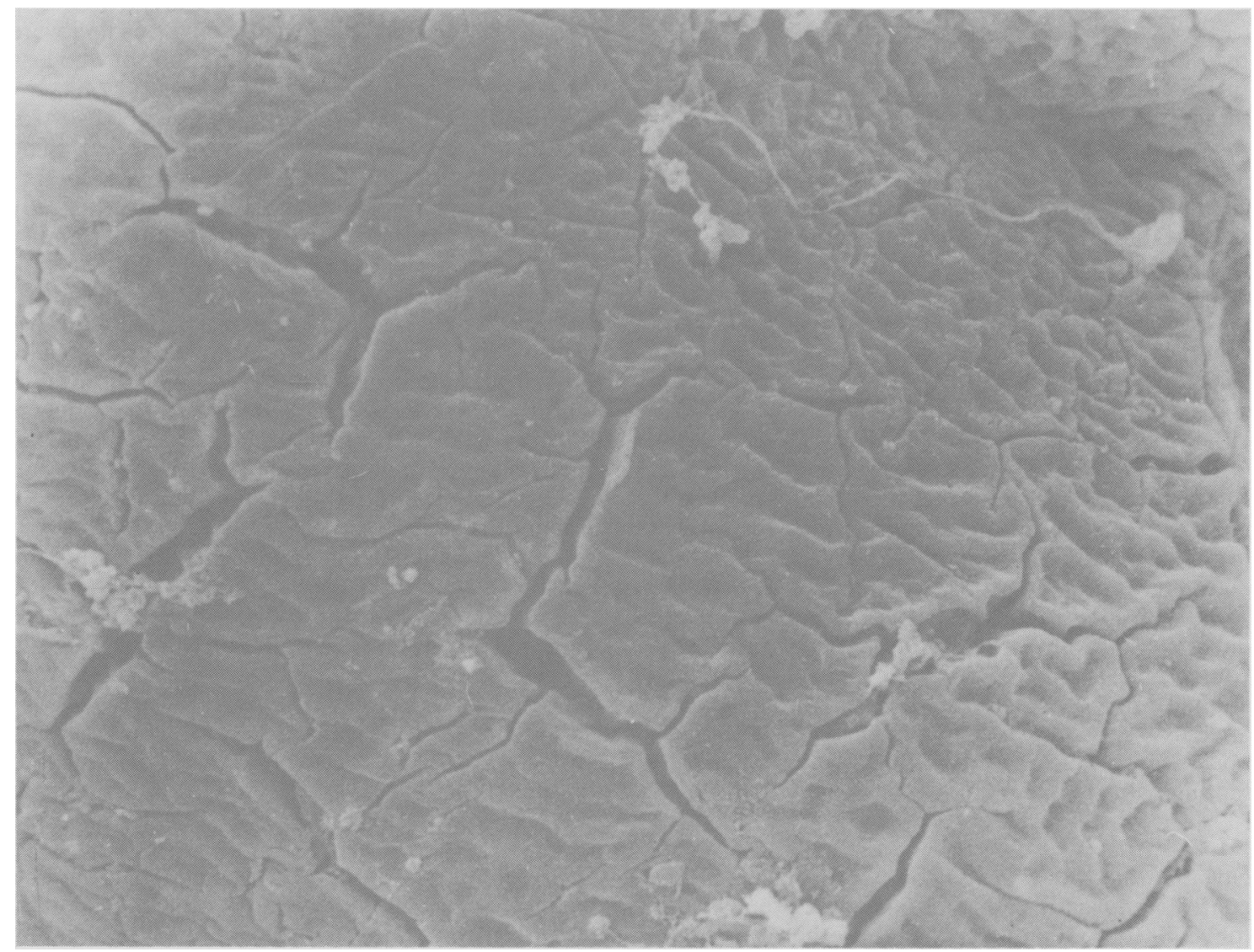

Fig. 2 Scanning electron microscopic appearances showing crypt units with wide orifices with swollen edges; the crypt unit integrity score was 8. 
Table 2 Total body weight change as a percentage of starting weight

\begin{tabular}{|c|c|c|c|}
\hline Diet & Group & $\begin{array}{c}\text { Measurement } \\
4 / I 2\end{array}$ & $\begin{array}{c}\text { Category } \\
6 / 12\end{array}$ \\
\hline \multirow[t]{2}{*}{1} & Control & $+13.9 \% *$ & $+2 \cdot 7 \%$ \\
\hline & AOM Treated & $-14.5 \% *$ & $+1 \cdot 8 \%$ \\
\hline \multirow[t]{2}{*}{2} & Control & $+13.3 \% *$ & $-25 \cdot 1 \% *$ \\
\hline & AOM Treated & $+1 \cdot 0 \%$ & $-18.4 \% *$ \\
\hline \multirow[t]{2}{*}{3} & Control & $+19 \cdot 2 \% *$ & $+29.3 \% *$ \\
\hline & AOM Treated & $-0.6 \%$ & $+2 \cdot 7 \%$ \\
\hline \multirow[t]{2}{*}{4} & Control & $+20.8 \% *$ & $+23 \cdot 1 \% *$ \\
\hline & AOM Treated & $+3.6 \%$ & $+11 \cdot 0 \% *$ \\
\hline
\end{tabular}

${ }^{*}$ Denotes a statistically significant change in weight.

comprising both neutral sterols and free faecal bile acids were analysed, the individual compounds being identified by computerised gas liquid chromatography and mass spectrometry.

\section{Results}

GENERAL CHARACTERISTICS

In total, $24(10 \cdot 34 \%)$ of the 232 animals failed to survive until the scheduled time of their death. Of these, six were in the control and 18 in the carcinogen treated groups. No pathological abnormalities were detected among the control groups and in the carcinogen treated animals, no deaths were clearly attributable to gastrointestinal neoplasia or any complications resulting therefrom. Considerable difficulty was encountered in achieving detailed post mortem information from these animals because of cannibalisation. The four different dietary groups in both the $0 / 12$ category and the $4 / 12$ category were not statistically different from one another with regard to either the weight of diet consumed or the calculated gross energy intake appropriate to that food intake. In the $6 / 12$ category both the weight of food taken in and the corresponding gross energy value was greater in animals fed diet 1 compared with all the other three dietary groups $(\mathrm{p}<0 \cdot 001)$.

Table 2 shows that the pattern amongst the four dietary groups for total body weight during the course of experiments was such that high fibre fed animals tended to thrive less well than low fibre fed animals. The only groups which consistently lost body weight were the 6/12 groups eating diet 2 (low fat, high fibre).

\section{PATHOLOGY}

No abnormalities were encountered in any of the control animals. Reliable autopsy data are available for $208(90 \%)$ animals. Among the carcinogen treated groups in the $4 / 12$ category the only neoplastic lesions noted were found among animals

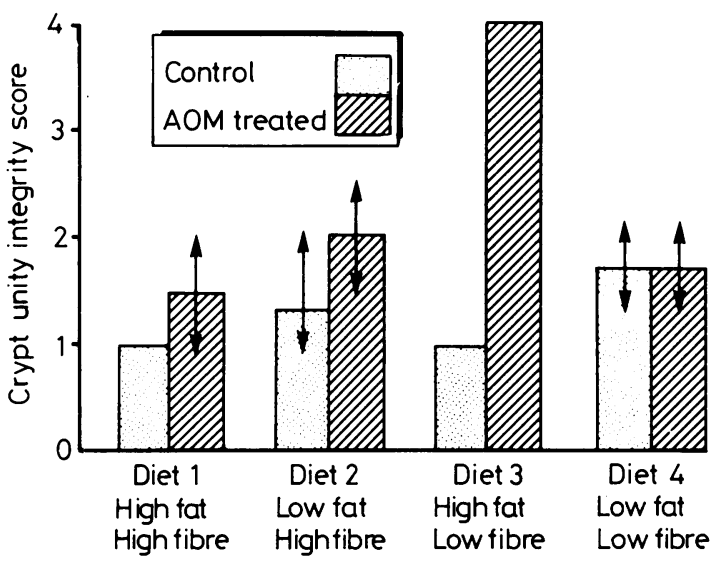

Fig. 3 Ultrastructural appearances. A crypt unit integrity score of one is regarded as normal. The mean and range for each group is shown where applicable.

consuming diet 3 (high fat, low fibre). Amongst these 10 animals, four had large bowel neoplasia; in two, lesions in the proximal colon were easily visible and in a further two microscopic adenomatous foci were detected on histological examination of mucosa which appeared macroscopically normal.

Within the 6/12 category an entire range of gastrointestinal neoplastic lesions was identified. The interdietary distribution of visible colonic tumours which were subsequently confirmed histologically was analysed by first comparing the median number of tumours in each dietary group using the KruskalWallis test (non-parametric analysis of variance). This revealed that the median number of tumours for each of the dietary groups showed statistically significant differences $(\mathrm{p}<0 \cdot 025)$. Follow up MannWhitney $U$ tests were carried out to compare each diet with each of the other diets. Diet 1 had significantly fewer tumours than diet $3(p<0.02)$ and diet $4(p<0 \cdot 05)$. Diet 2 had significantly fewer tumours than diet $3(p<0.02)$ and diet $4(p<0.05)$. Finally, diet 3 animals had significantly more tumours than diet 4 counterparts.

In total, 55 gastrointestinal neoplasms were confirmed among all the carcinogen treated groups in these experiments. Six $(10.9 \%)$ of these lesions were found in animals fed diet 1 . Two $(3 \cdot 6 \%)$ of the lesions occurred in animals fed diet 2 (low fat, high fibre). In contrast, $35(63.7 \%)$ occurred in high fat, low fibre fed animals, - that is, diet 3, and the remaining 12 $(21.8 \%)$ were found in the low fat, low fibre fed group.

Careful assessment of crypt length showed no evidence of any significant differences between any carcinogen treated groups and their corresponding controls. Thus in this study there is no direct evidence of any azoxymethane induced crypt hyperplasia. 


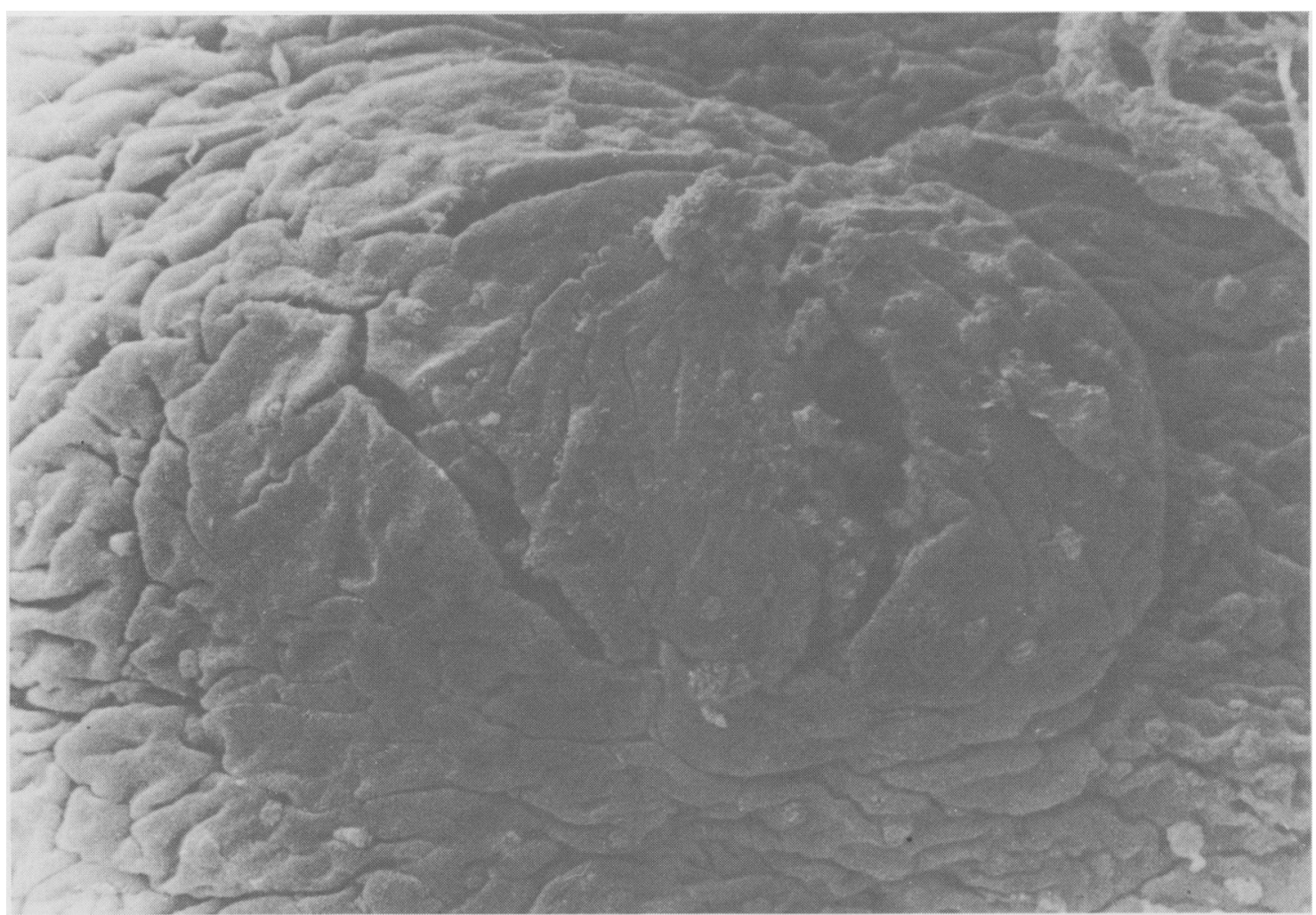

Fig. 4 Scanning electron microscopic appearances of tiny adematous focus involving several adjacent crypts.

TOPOGRAPHICAL CHARACTERISTICS

No major topographical changes can be attributed to diet along the colon, although slight differences in surface architecture were apparant between the dietary groups, with the highest crypt unit integrity score applying to animals fed diet 2 (low fat, high fibre). After carcinogen treatment a much greater degree of deviation from the normal pattern was seen. These changes were most severe in the animals fed diet 3 (high fat, low fibre), see Figure 3.
Figure 4 shows an example of the scanning electron microscopic appearances of a lesion considered to represent early colonic neoplasm.

CELL POPULATION KINETICS

Table 3 shows the values for the crypt cell production rates (CCPR) in the animals in the 4/12 category. There was considerable variability within those data and no clear pattern exists with respect to an effect of any of the major variables, namely diet, anatomical

Table 3 Crypt cell production rate (cells/crypt/hour) 4/12 category: control and carcinogen treated groups

\begin{tabular}{|c|c|c|c|c|c|c|c|c|}
\hline \multirow{3}{*}{ Diet } & \multicolumn{8}{|c|}{ Anatomical site } \\
\hline & \multicolumn{2}{|c|}{ Rectum } & \multicolumn{2}{|c|}{ Descending colon } & \multicolumn{2}{|c|}{ Transversecolon } & \multicolumn{2}{|c|}{ C'aecum } \\
\hline & Control & AOM & Control & $\mathrm{AOM}$ & Control & $\mathrm{AOM}$ & Control & $\mathrm{AOM}$ \\
\hline \multicolumn{9}{|l|}{1 High fat } \\
\hline High fibre & $7 \cdot 54$ & $7 \cdot 82$ & $12 \cdot 72$ & $12 \cdot 57$ & $12 \cdot 71$ & 9.55 & 8.91 & $9 \cdot 69$ \\
\hline \multicolumn{9}{|l|}{2 Low fat } \\
\hline High fibre & $11 \cdot 52$ & $11 \cdot 16$ & $4 \cdot 82$ & $21 \cdot 51$ & 13.45 & $42 \cdot 47$ & $12 \cdot 69$ & $14 \cdot 50$ \\
\hline \multicolumn{9}{|l|}{3 High fat } \\
\hline Low fibre & $4 \cdot 23$ & $3 \cdot 32$ & 3.69 & $6 \cdot 72$ & $7 \cdot 80$ & $8 \cdot 23$ & $7 \cdot 81$ & $5 \cdot 21$ \\
\hline \multicolumn{9}{|l|}{4 Low fat } \\
\hline Low fibre & $9 \cdot 25$ & $7 \cdot 32$ & $14 \cdot 10$ & $10 \cdot 40$ & $12 \cdot 60$ & $11 \cdot 0(0)$ & 8.55 & $x \cdot 48$ \\
\hline
\end{tabular}


Fig. 5 Summary of statistical analysis of stathmokinetic data using GLIM and a weighted one way analysis of variance. Multiple comparisons were performed and the significant interdietary differences are displayed in the grid.

site and the use of azoxymethane. A weighted one-way analysis of variance has been carried out using the generalised linear interactive modelling technique in an attempt to do multiple comparisons between the different dietary groups and discern any significant pattern which might exist in the data.

The significant differences generated are shown in Figure 5 which shows that there was no clear or consistent effect of any individual diet on cell kinetic parameters. In the $0 / 12$ category the only significant dietary influence in kinetic parameters occurred in the most distal portion of the bowel. Fibre seems to have a more pronounced individual effect than fat, the tendency being for the diets containing high levels of fibre to be associated with more rapid cell proliferation than those containing less fibre.

In the $4 / 12$ control category significant dietary influences were again seen in the distal colorectum. Here, however, attempts to distinguish individual effects of fat and fibre yield conflicting impressions and the results appear inconsistent. In the 4/12 carcinogen treated animals the most interesting and consistent contrasts appear. Dietary effects can be seen at each of the anatomical sites examined. For the rectum the individual fat and fibre effects are such that in the significant differences which do appear, high fat is associated with slower CCPR and high fibre with faster CCPR. For the descending and transverse colon exactly the same individual nutrient effects can be seen and reinforcing this, in each

instance the low fat high fibre diet (diet 2) was associated with significantly faster CCPR than the high fat low fibre diet (diet 3). In the caecum no significant individual effect of fat can be identified but, once again, increased dietary fibre was associated with faster CCPR.

In the $6 / 12$ control animals the kinetic activity is rather more even and no significant dietary differences appeared. In the corresponding carcinogen treated animals there was only one isolated difference between diets 1 and 4 .

\section{FAECAL CHARACTERISTICS}

PHYSICAL PROPERTIES

Table 4 shows the weight of faecal output for control and carcinogen treated groups, during both week six and week 16 of the study, averaged and expressed as grams of faeces per rat per 24 hours. High fibre containing diets (diets 1 and 2) were associated with a much more marked bulking effect than the low fibre containing diets $(p<0 \cdot 001)$. Furthermore no significant differences were seen which could be attributed to dietary fat or the administration of carcinogen.

\section{FAECAL OCCULT BLOOD}

In 208 animals Haemoccult tests were carried out and $22(10 \cdot 57 \%)$ were positive. Sensitivity and specificity have been calculated with respect to macroscopically obvious gastrointestinal lesions which subsequently were confirmed to be neoplastic on histological examination. There were five false positive slides and one false negative. Thus the test had a sensitivity of $94.4 \%$ and a specificity of $97.3 \%$ The predictive accuracy was $77.2 \%$ for a positive result and $99.4 \%$ for a negative result.

\section{FAECAL BILE ACID CONCENTRATION}

Figure 6 shows a summary of the total faecal free bile acid (FBA) concentration expressed as milligrams per gram dry faeces. The total FBA concentration in dietary groups 1 and 2 , the high fibre containing diets,

Table 4 Faecal weight (g/rat/day) as measured during two separate periods for groups in the 4/12 category

\begin{tabular}{|c|c|c|c|c|}
\hline \multirow[t]{2}{*}{ Diet } & \multicolumn{2}{|c|}{ Control groups } & \multicolumn{2}{|c|}{ Carcinogen treated groups } \\
\hline & Week 6 & Week 16 & Week 6 & Week 16 \\
\hline $\begin{array}{l}1 \text { High fat } \\
\text { High fibre }\end{array}$ & $12 \cdot(03$ & $12 \cdot 25$ & $11 \cdot 75$ & $9 \cdot 98$ \\
\hline $\begin{array}{l}2 \text { Low fat } \\
\text { High fibre }\end{array}$ & $12 \cdot 25$ & 11.95 & $11 \cdot 82$ & $14 \cdot 00$ \\
\hline $\begin{array}{l}3 \text { High fat } \\
\text { Low fibre }\end{array}$ & $1 \cdot 39$ & $1 \cdot 88$ & $1 \cdot 35$ & 1.82 \\
\hline $\begin{array}{l}4 \text { Low fat } \\
\text { Low fibre }\end{array}$ & $2 \cdot 01$ & $1 \cdot 40$ & $1 \cdot 90$ & $1 \cdot 32$ \\
\hline
\end{tabular}



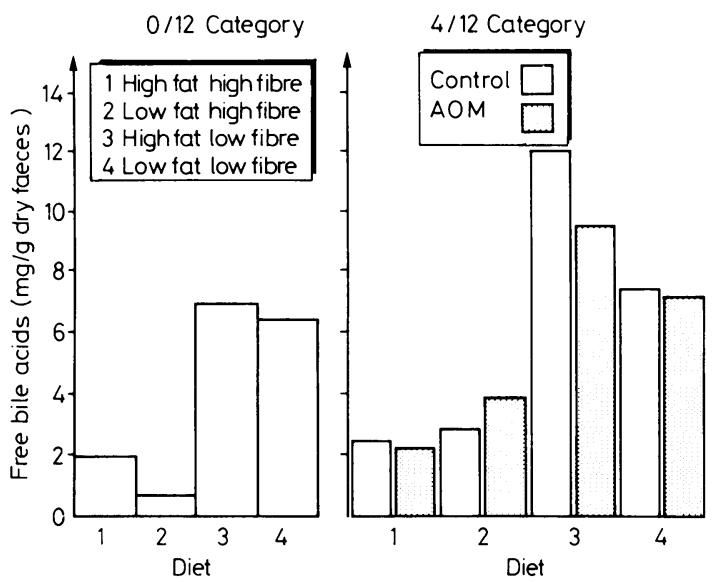

Fig. 6 Faecal free bile acid concentration ( $\mathrm{mg} / \mathrm{g}$ dry faeces); $0 / 12$ and 4/12 categories.

was considerably lower than the corresponding low fibre containing diets 3 and 4 where the values were from 3.4-10.9 times greater. Neither dietary fat nor the administration of azoxymethane produced a significant or consistent effect on FBA concentration.

\section{Discussion}

Despite the many mechanisms postulated by which dietary fat and fibre may exert promoting and protecting influences on colorectal carcinogenesis, the exact importance of each and their inter relationships are still poorly understood.

\section{TUMOUR INDUCTION}

In this study the two techniques of histology and SEM show that both the levels of dietary fat and fibre have a bearing on the ultimate tumour risk applying to carcinogen treated animals consuming any one of the four diets. As in other investigations ${ }^{1+17}$ high levels of fat enhanced carcinogenesis, and high levels of fibre ${ }^{1 \mathrm{x}-14} \mathrm{had}$ a potent protective effect. In addition there is good evidence of a significant interaction between the promoting and protecting influences of fat and fibre.

The deliberate variation of any single dietary component will require the addition or displacement of other components thus altering the relative composition of at least two dietary variables. The role of other crude nutrients such as protein and carbohydrate on subsequent tumour induction is seen here to be of little importance. The protein content of the four study diets is closely similar despite widely differing risks for tumour development. While carbohydrate levels differed between the groups they were most closely similar between diets 2 and 3, for which tumour induction patterns were most dissimilar. Neither protein nor carbohydrates have been previously thought to influence carcinogenesis significantly. There is no direct relationship between tumour induction and food or energy intake in this study. It is possible, however, that the protective effect of diet 2 (low fat, high fibre) may have been accentuated by the failure of those particular groups to thrive.

Current understanding of the role of dietary factors on intestinal carcinogenesis implicates the metabolic activity of the bacterial population of the gastrointestinal tract. The flora can be altered by changes in dietary practices." Whether fibre produces its effect by some direct physicochemical action or by increasing faecal bulk, thereby diluting and possibly minimising any contact between the mucosa, and as yet unidentified carcinogens, is unknown. ${ }^{21}$ In this study fibre was the only nutrient associated with any significant alteration in faecal bulk.

The proposed promoting mechanism for fat has been closely linked with faecal bile acid concentration. It has been shown that in man, increasing the dietary intake of fat was associated with an increase in FBA concentration. In experimental animals, secondary bile acids have cocarcinogenic properties. While in the present study a comparison of the diets with respect to FBA concentration showed a pattern which paralleled that of tumour induction, the major dietary determinant of FBA concentration was fibre rather than fat content.

DYNAMIC CELL POPULATION KINETICS

While the fundamental relevance of cell kinetic studies to colorectal neoplasia is not in dispute, the actual kinetic processes involved in tumour development are more controversial." Dietary manipulation in this study produced no overall clear-cut kinetic effect. Carcinogen treated groups in the preneoplastic phase of tumour development (4/12 category) did not show some striking fat and fibre related trends in colonic proliferative activity. The slower kinetic activity tended to occur in dietary groups at high risk for tumour induction, the lower risk diet groups having more rapid cell production rates. High fibre containing diets were frequently associated with fast cell production, while high fat, in contrast, was often linked to slower cell proliferation. There were no kinetic effects attributable to individual factors such as azoxymethane, protein, carbohydrate, energy intake or body weight.

In view of the presence of discernable diet related kinetic effects at only one stage of carcinogenesis it may be postulated that these proliferative patterns are relevant to subsequent tumour production at one crucial part of a complex multistage process. Much 
evidence is now available to support the concept that rapid proliferative activity present at the time of initiation enhances carcinogenesis. ${ }^{2+2.20}$ This study is the first to report proliferative differences related to dietary promoting and protecting factors during the postinitiation phase. A study ${ }^{27}$ in which dietary fibre has been manipulated, however, has suggested that the hyperproliferative effect of fibre has an enhancing effect on tumour initiation but a protective effect during promotion. No hyperplasia after azoxymethane treatment was noted in this study. Sunter $^{2 *}$ did find such hyperplasia in a closely similar experimental model and it seemed to be accompanied by a rise in cell production rate as carcinogenesis progressed. Cooke ${ }^{2 y}$ also noted progressively increasing crypt cell production rates as neoplasia progressed, although it is of interest that the tumour distribution in their study corresponded to the areas where crypt cell production rate had been slowest during the early stages of promotion.

The work reported here together with that of Tutton and Barkla ${ }^{311}$ appears to conflict with the suggestion that an increased cell proliferation rate is a fundamental kinetic process during tumour induction. Indeed these latter studies emphasise the importance of a slowly proliferating cell population to the evolution of colorectal neoplasia. Specific slowing of cell proliferation within an initiated cell population may allow those cells more time within the mucosa to establish their selective growth advantage. Given that tumour development probably involves many stages it may be that all these kinetic observations are not incompatible, but reflect measurements made at different parts of a complex process. The relationship between cell kinetics, tumour promotion and histogenesis requires further study to determine more accurately whether the cell kinetic differences seen in this study are crucial or epiphenomenal.

\section{References}

1 Wynder EL, Shigematsu T. Environmental factors in cancer of the colon and rectum. Cancer 1967; 23: $1520-61$.

2 Correa P, Haenszel W. The epidemiology of large bowel cancer. Adv Cancer Res 1978; 26: 1-141.

3 Berg J, Howell MA. The geographic pathology of large bowel cancer. Cancer 1974; 134: 807-14.

4 Burkitt DP. Relationship has a clue to causation. Lancet 197(); 2: 1237-4().

5 LaMont JT, O'Gorman TA. Experimental colon cancer. Gastroenterology 1978; 75: 1157-69.

6 Morson BC, Bussey HJR, Day DW, Hill MJ. Adenoma of large bowel Cancer Surv 1983; 2: 451-77.

7 Shamsuddin AKN, Trump BJ. Colon epithelium. II In vivo studies of colon carcinogenesis. Light microscopic, histochemical and ultrastructural studies of histogenesis of azoxymethane induced colon carcinomas in Fisher 344 rats. J Nat Cancer Inst 1981; 66: 389-3.

8 Lipkin M. Phase 1 and Phase 2 proliferative lesions of colonic epithelial cells in diseases leading to colon cancer. Cancer 1974: suppl 34.

9 Wright NA and Appleton DR. The metaphase arrest technique. A critical review. Cell Tissue Kinet 1980; 13: 643-63.

10 Carr KE, McLay ALC, Toner PG, Chung P, Wong A. Scanning electron-microscopy in service pathology. A review of its potential role. Scan Electron Microsc 1980; 3: $121-38$

11 Carr KE, Hamlet R, Niaz AHW, Watt C. Damage to the surface of the small intestinal willus: an objective scale of assessment on the effects of single and fractionated radiation doses. Br J Radiol 1983; 56: 467-75.

12 Baker RJ, Nelder JA. The Glim system. Generalised linear interactive modelling. London: Royal Statistical Society 1978.

13 Owen RW, Thompson MH, Hill MJ. Analysis of metabolic profiles of steroids in faeces of healthy subjects undergoing chenodeoxycholic acid treatment by liquid - gel chromatography and gas - liquid chromatography - mass spectrometry. J Steroid Biochem 1984; 21: 593-600.

14 Broitman SA, Vitale JJ, Vavronsek-Jakuta LS. Polyunsaturated fat, cholesterol and large bowel tumorogenesis. Cancer 1977; 40: 2455-63.

15 Nigro ND, Singh DV, Campbell RL, Sook M. Effects of dietary beef fat on intestinal tumour formation by azoxymethane in rats. $J$ Nat Cancer Inst 1975; 54: 439-42.

16 Reddy BS, Narisawa T, Vukusich D, Weisburger JH, Wynder EL. Effect of quality and quantity of dietary fat and dimethylhydrazine colon carcinogenesis in rats. Proc Soc Exp Biol Med. 1976; 151: 237-9.

17 Trudel JL, Senterman MK, Brown RA. The fat/fibre antagonism in experimental colon carcinogenesis. Surgery 1983; 94: 691-6.

18 Fleiszer DM, Murrey D, Richards GL, Brown RA. Effects of diet on chemically induced bowel cancer. Can J Surg 1980; 23: 67-74.

19 Freeman HJ, Spiller GA, Kim YS. A double blind study on the effect of purified cellulose dietary fibre on 1 , 2 dimethylhydrazine induced colonic neoplasia. Cancer Res 1978; 38: 2912-7.

20 Aries VC, Crowther JS, Draser BS, Hill Mj, Williams REO. Bacteria and the aetiology of cancer of the large bowel. Gut 1969; 10: 334-5.

21 Freeman HJ. Dietary fibers and colon cancer. In: Autrup $\mathrm{H}$ and Williams GM, eds. Experimental Colon Carcinogenesis Boca Raton: CRC Press, 1983.

22 Hill MJ. The effect of some factors on the faecal concentration of acid steroids, neutral steroids and urobilins. J Pathol 1971; 104: 239-45.

23 Wright NA. The Histogenesis of gastrointestinal cancer. Hodgson HJF, Bloom SR eds. Gastrointestinal and hepatobiliary cancer. London: Chapman and Hall, 1983.

24 Pozharrisski KM. The significance of non-specific injury for colon carcinogenesis in rats. Cancer Res 1975; 35: 3824-30.

25 Barthold SW, Beck D. Modification of early dimethyl- 
hydrazine carcinogenesis by colonic mucosal hyperplasia. Cancer Res 1980; 40: 4451-5.

26 Williamson RCN, Davis PW, Bristol JB, Wells M. Intestinal adaptation and experimental carcinogenesis after partial colectomy. Gut 1982; 23: 316-25.

27 Jacobs LR. Enhancement of rat colon carcinogenesis by wheat bran consumption during the stage of 1,2 dimethylhydrazine administration. Cancer Res 1983;43: 4057-61.

28 Sunter JP. Experimental carcinogenesis and cancer in the rodent gut. In: Appleton DR, Sunter JP, Watson
AJ, eds. Cell proliferation in the gastrointestinal tract. London: Pitman Medical, 1980.

29 Cooke T, Kirkham N, Stainthorp DH, Inman C, Goeting N, Taylor I. Detection of early neoplastic changes in experimentally induced colorectal cancer using scanning electron microscopy and cell kinetic studies. Gut 1984; 25: 748-55.

30 Tutton PJM, Barkla DH. Cell proliferation in the descending colon or dimethylhydrazine treated rats and in dimethylhydrazine induced adenocarcinomas. Virchows Archiv [Cell Pathol] 1976; 21 : 147. 